\title{
Idiopathische Lungenfibrose - Quiz zur Zertifizierung
}

\section{Nur eine der jeweiligen fünf Antwortmöglichkeiten ist richtig!}

1. Typische Symptome, die am ehesten an eine idiopathische Lungenfibrose denken lassen, sind
A: Luftnot, produktiver Husten
B: progressive Belastungsdyspnoe, trockener Husten
C: Thoraxschmerzen, Luftnot, Hämoptysen
D: nächtliche Atemnotanfälle, trockener Husten
E: Fieber, akut progrediente Luftnot

2. Welche der Aussagen zur Diagnostik der idiopathischen Lungenfibrose trifft zu?

A: Das HR-CT ist in der Diagnostik verzichtbar

B: Ein unauffälliges Thorax-Röntgenbild schließt eine idiopathische Lungenfibrose aus

C: Im HR-CT kann mit hoher Spezifität die Diagnose gestellt werden

D: Eine transbronchiale Lungenbiopsie ist der Goldstandard in der morphologischen Diagnostik

E: Bei fortgeschrittener Erkrankung finden sich milchglasartige Trübungen im HR-CT

3 In der Differenzialzytologie einer bronchoalveolären Lavage bei Patienten mit einer idiopathischen Lungenfibrose finden sich
A: $60 \%$ Lymphozyten
B: $20 \%$ neutrophile Granulozyten
C: $80 \%$ neutrophile Granulozyten
D: $40 \%$ eosinophile Granulozyten
E: $90 \%$ Makrophagen

4 Welche Untersuchungsmethode ist die sensitivste in der Erfassung einer Lungenfunktionsstörung bei idiopathischer Lungenfibrose?
A: Spirometrie
B: Spiroergometrie
C: Bestimmung des Gastransfers (DLCO)
D: Blutgasanalyse
E: Bestimmung der Lungencompliance

5 Ein Kriterium der nicht-spezifischen interstitiellen Pneumonie ist

A: ein Röntgenbefund mit konfluierenden Verschattungen

B: Honigwabenbildung im hochauflösenden CT

C: das Auftreten ausschließlich bei Rauchern

D: die Erstmanifestation in fortgeschrittenem Alter

E: die vergleichsweise günstige Prognose
6 Die Therapie der idiopathischen Lungenfibrose ist

A: durch kontrollierte Studien gesichert

B: nach den Kriterien der "Evidence-based Medicine" auszurichten

C: von einer internationalen Konsensuskonferenz aktuell empfohlen worden

D: empirisch durchzuführen

E: nach den eindeutigen validierten primären Endpunkten auszurichten

\section{Die gesicherte Lungenfibrose muss behandelt werden}
A: in jedem Fall
$B$ : in keinem Fall
C: eine abwartende Beobachtung ist möglich
D: nur in Abhängigkeit vom histologischen Befund
E: nur in Abhängigkeit von der Lungenfunktion

8 Als gesichert wirksame antifibrotisch aktive Substanzen können gelten
A: Suramin
B: Interferon $\gamma$
C: keine Substanz
D: Pirferidon
E: Interferon $\beta$

9 Die zurzeit in einer Konsensuskonferenz empfohlene Therapie besteht aus
A: Kortikosteroiden
B: Pirferidon
C: Azathioprin
D: Prednisolon plus Azathioprin
E: Cyclosporin A

10 Die Ergebnisse der bronchoalveolären Lavage können therapeutisch hilfreich sein
A: in jedem Fall
B: nein
C: bei der Monotherapie
D: bei der Kombinationstherapie
E: bei der Mono- und bei der Kombinationstherapie

\section{Prognostisch günstige Ausgangsbefunde sind}
A: hohes Alter
B: männliches Geschlecht
C: kurzer, unter 1 Jahr liegender Symptomverlauf
D: zystische Veränderungen im HR-CT
E: Abnahme des proportionalen Lymphozytenanteils in der bronchoalveolären Lavage 